\title{
DYNAMICS OF GLOBAL ATTRACTOR FOR A SEMILINEAR DEGENERATE PARABOLIC EQUATION INVOLVING GRUSHIN OPERATORS
}

\author{
JIHOON LEE \\ Department of Mathematics \\ Yonsei University Seoul, 03722, KOREA
}

\begin{abstract}
In this paper we study the dynamics of global attractor of a semilinear parabolic equation involving Grushin operators. First we show that the global attractor is bounded in $L^{\infty}(\Omega)$ and $D(A)$. Then we investigate the existence of a Lyapunov function, the injectivity on the global attractor and the squeezing property. Finally, we obtain estimates on upper bound and lower bound of the fractal dimension of the global attractor.
\end{abstract}

AMS Subject Classification: 35J70, 35B05

Key Words: global attractor, Grushin operator, Lyapunov function, injectivity, squeezing property, determining modes, fractal dimension

Received: September 2, 2018; Accepted: March 18, 2018;

Published: June 7, $2018 \quad$ doi: 10.12732/dsa.v27i3.2

Dynamic Publishers, Inc., Acad. Publishers, Ltd. https://acadsol.eu/dsa

\section{INTRODUCTION}

One of the most important problems in modern mathematical physics is to understand the asymptotic behavior of the trajectories of infinite-dimensional dynamical systems induced by PDEs. One way to investigate the problem for dissipative dynamical systems is to study their global attractors which contain much of the relevant information about the systems and often some finite-dimensional characters. The first step is to study the existence of a global attractor. After that, we study the dynamics of the global attractor such as the regularity, structure, continuous dependence on parameters, fractal dimension estimates, etc. 
Recently, the class of equations involving an operator of Grushin type

$$
G_{s} u=\Delta_{x} u+|x|^{2 s} \Delta_{y} u, \quad s \geq 0
$$

has been studied widely by many mathematicians (for examples, see $[1,2,4,5,6$, $10,11,14,15,16])$. Here $(x, y) \in \Omega$ and $\Omega$ is a bounded domain in $\mathbb{R}^{N}=\mathbb{R}^{N_{1}} \times$ $\mathbb{R}^{N_{2}}\left(N_{1}, N_{2} \geq 1\right)$ with smooth boundary $\partial \Omega$. The operator $G_{s}$ was first introduced by Grushin in [7] when $s$ is a positive integer, and later by Franchi and Lanconelli in [6] when $s$ is not an integer. Note that $G_{0}$ is the Laplace operator denoted by $\Delta$, and $G_{s}$, when $s>0$, is not elliptic (but hypoelliptic) in domains of $\mathbb{R}^{N_{1}} \times \mathbb{R}^{N_{2}}$ intersecting the surface $\{x=0\}$.

In this paper, we study the dynamics of the global attractor for the following semilinear degenerate parabolic problem

$$
\begin{cases}u_{t}-G_{s} u+f(u)=g(x), & x \in \Omega, t>0, \\ u(x, t)=0, & x \in \partial \Omega, t>0, \\ u(x, 0)=u_{0}(x), & x \in \Omega .\end{cases}
$$

Note that if $s=0$, the equation (1.1) is just the nonlinear reaction-diffusion equation. Here we assume that $u_{0} \in L^{2}(\Omega), g \in L^{\infty}(\Omega)$, and $f: \mathbb{R} \rightarrow \mathbb{R}$ is a function of polynomial type, that is, $f$ is a $C^{1}$ function satisfying the following conditions

$$
\begin{gathered}
c_{1}|s|^{p}-c_{0} \leq f(s) s \leq c_{2}|s|^{p}+c_{0}, \quad p \geq 2, \\
f^{\prime}(s) \geq-\ell
\end{gathered}
$$

where $c_{0}, c_{1}, c_{2}$ and $\ell$ are positive constants. A typical example of such functions is a polynomial of odd order with positive leading coefficient

$$
f(u)=\sum_{i=0}^{2 p+1} a_{i} u^{i}, \quad a_{i} \in \mathbb{R}, \quad a_{2 p+1}>0 .
$$

The existence and uniqueness of weak solutions, and the existence of a global attractor $\mathcal{A}$ in $L^{2}(\Omega)$ of the continuous semigroup $S(t)$ generated by (1.1) were proved by Anh and Ke in [2] under the assumption $g \in L^{2}(\Omega)$.

To study problem (1.1), we recall some weighted function spaces which will be used in the paper. Let $S_{0}^{1}(\Omega)$ be the completion of $C_{0}^{1}(\bar{\Omega})$ with the norm

$$
\|u\|_{S_{0}^{1}}=\left(\int_{\Omega}\left|\nabla_{x} u\right|^{2}+|x|^{2 s}\left|\nabla_{y} u\right|^{2} d x d y\right)^{\frac{1}{2}} .
$$

Then we know that $S_{0}^{1}(\Omega)$ is a Banach space and the embedding $S_{0}^{1}(\Omega) \hookrightarrow L^{2}(\Omega)$ is compact. Denote $A=-G_{s}$ with the homogeneous Dirichlet boundary condition. Then its domain

$$
D(A)=\left\{u \in S_{0}^{1}(\Omega) \mid A u \in L^{2}(\Omega)\right\}
$$


is a Banach space with the graph norm

$$
\|u\|_{D(A)}=\left(\int_{\Omega}\left|G_{s} u\right|^{2} d x d y\right)^{\frac{1}{2}} .
$$

We also know that the embedding $D(A) \hookrightarrow S_{0}^{1}(\Omega)$ is compact.

The paper is organized as follows. In Section 2 , we show that the global attractor of the semigroup $S(t)$ induced by (1.1) is bounded in $L^{\infty}(\Omega)$ and $D(A)$. In Section 3, we investigate the existence of a Lyapunov function, the injectivity on the global attractor, the squeezing property and its consequences. In Section 4, we obtain estimates on upper bound and lower bound of the fractal dimension of the global attractor. These are an extension of some previous results for the nonlinear reaction-diffusion equations in $[9,12,13]$.

Throughout the paper, for brevity, we denote by $|\cdot|,\|\cdot\|_{\infty}$ and $\|\cdot\|_{S_{0}^{1}}$ the norms in the spaces $L^{2}(\Omega), L^{\infty}(\Omega)$ and $S_{0}^{1}(\Omega)$, respectively.

\section{BOUNDEDNESS OF GLOBAL ATTRACTORS}

In this section, we prove that the global attractor $\mathcal{A}$ of the semigroup $S(t)$ induced by (1.1) is bounded in both $L^{\infty}(\Omega)$ and $D(A)$, which will be used in the next sections.

Theorem 2.1. The global attractor $\mathcal{A}$ of the equation $(1.1)$ is bounded in $L^{\infty}(\Omega)$.

Proof. For $u \in L^{2}(\Omega)$, we denote

$$
u_{+}(x, y)= \begin{cases}u(x, y), & u(x, y)>0 \\ 0, & \text { otherwise }\end{cases}
$$

and similarly

$$
u_{-}(x, y)= \begin{cases}u(x, y), & u(x, y)<0 \\ 0, & \text { otherwise }\end{cases}
$$

By (1.2), we have

$$
f(s) s \geq c_{1}|s|^{p}-c_{0}
$$

for all $s \in \mathbb{R}$. Hence we get

$$
f(s) \geq\|g\|_{\infty} \text { when } s \geq M \text { for sufficiently large } M>0 \text {. }
$$

Multiplying the first equation in $(1.1)$ by $(u-M)_{+}$and integrating over $\Omega$ yield

$$
\frac{1}{2} \frac{d}{d t} \int_{\Omega}(u-M)_{+}^{2} d x d y+\int_{\Omega}\left(\left|\nabla_{x}(u-M)_{+}\right|^{2}+|x|^{2 s}\left|\nabla_{y}(u-M)_{+}\right|^{2}\right) d x d y
$$




$$
+\int_{\Omega} f(u)(u-M)_{+} d x d y=\int_{\Omega} g(u-M)_{+} d x d y
$$

By (2.1), we see that

$$
\frac{1}{2} \frac{d}{d t} \int_{\Omega}(u-M)_{+}^{2} d x d y+\int_{\Omega}\left(\left|\nabla_{x}(u-M)_{+}\right|^{2}+|x|^{2 s}\left|\nabla_{y}(u-M)_{+}\right|^{2}\right) d x d y \leq 0 .
$$

Since the space $S_{0}^{1}(\Omega)$ is embedded into $L^{2}(\Omega)$, we have

$$
\int_{\Omega}\left(\left|\nabla_{x}(u-M)_{+}\right|^{2}+|x|^{2 s}\left|\nabla_{y}(u-M)_{+}\right|^{2}\right) d x d y \geq C \int_{\Omega}(u-M)_{+}^{2} d x d y .
$$

It follows that

$$
\frac{d}{d t} \int_{\Omega}(u-M)_{+}^{2} d x d y \leq-C \int_{\Omega}(u-M)_{+}^{2} d x d y
$$

and so

$$
\int_{\Omega}(u-M)_{+}^{2} d x d y \leq e^{-C t} \int_{\Omega}\left(u_{0}-M\right)_{+}^{2} d x d y .
$$

Since $\mathcal{A}$ is bounded in $L^{2}(\Omega)$ (Theorem 2.6 in [2]) and invariant under $S(t)$, we obtain

$$
\int_{\Omega}(u-M)_{+}^{2} d x d y=0
$$

for all $u \in \mathcal{A}$. We apply a similar argument to $(u+M)_{-}$to deduce that

$$
\int_{\Omega}(u+M)_{-}^{2} d x d y=0
$$

for all $u \in \mathcal{A}$. This shows that

$$
\|u\|_{\infty} \leq M \text { for all } u \in \mathcal{A} \text {. }
$$

Using the boundedness of $\mathcal{A}$ in $L^{\infty}(\Omega)$, we now show that $\mathcal{A}$ is bounded in $D(A)$.

Theorem 2.2. The global attractor $\mathcal{A}$ of the equation (1.1) is bounded in $D(A)$.

Proof. We rewrite the equation (1.1) as

$$
A u=-\frac{d u}{d t}-f(u)+g, \quad u(0)=u_{0} .
$$

To prove the theorem, it is enough to show that the right-hand side of the first equation of $(2.2)$ is bounded in $L^{2}(\Omega)$ on $\mathcal{A}$. Since $u \in L^{\infty}(\Omega)$, we see that $f(u) \in L^{2}(\Omega)$, and we have had $g \in L^{2}(\Omega)$.

First let us estimate $\int_{0}^{t}\left|u_{t}\right|^{2} d s$. Multiply $u_{t}:=\frac{d}{d t} u$ to both sides of the following equation

$$
u_{t}+A u+f(u)=g .
$$


Then we get

$$
\left|u_{t}\right|^{2}+\frac{1}{2} \frac{d}{d t}\|u\|_{S_{0}^{1}}^{2}+\int_{\Omega} f(u) u_{t} d x d y=\int_{\Omega} g(x, y) u_{t} d x d y
$$

and so

$$
\left|u_{t}\right|^{2}+\frac{1}{2} \frac{d}{d t}\|u\|_{S_{0}^{1}}^{2}+\frac{d}{d t} \int_{\Omega} F(u) d x d y=\frac{d}{d t} \int_{\Omega} g(x, y) u d x d y
$$

where $F(s)=\int_{0}^{s} f(\sigma) d \sigma$ is a primitive of $f$. Integrating the equality (2.4) from 0 to $t$ gives

$$
\begin{aligned}
\int_{0}^{t}\left|u_{t}\right|^{2} d t+\frac{1}{2}\|u(t)\|_{S_{0}^{1}}^{2}= & \frac{1}{2}\left\|u_{0}\right\|_{S_{0}^{1}}^{2}-\int_{\Omega} F(u(x, y, t)) d x d y+\int_{\Omega} F(u(x, y, 0)) d x d y \\
& +\int_{\Omega} g(x, y) u(x, y, t) d x d y-\int_{\Omega} g(x, y) u(x, y, 0) d x d y .
\end{aligned}
$$

Since $\mathcal{A}$ is bounded in both $S_{0}^{1}(\Omega)$ and $L^{\infty}(\Omega)$, we see that

$$
\int_{0}^{t}\left|u_{t}\right|^{2} d t+\frac{1}{2}\|u(t)\|_{S_{0}^{1}}^{2} \leq K
$$

for some $K>0$.

Next we obtain the bound on $u_{t}$ in $L^{2}(\Omega)$. Differentiate (2.3) to obtain

$$
\frac{d}{d t} u_{t}+A u_{t}+f^{\prime}(u) u_{t}=0
$$

If we take the inner product with $t^{2} u_{t}$, then we get

$$
\left(t^{2} u_{t}, \partial_{t} u_{t}\right)-t^{2}\left(u_{t}, G_{s} u_{t}\right)=-t^{2}\left(f^{\prime}(u) u_{t}, u_{t}\right) .
$$

Noting that $f^{\prime}(s) \geq-\ell$ for all $s \in \mathbb{R}$, it follows that

$$
\frac{1}{2} \frac{d}{d t}\left|t u_{t}\right|^{2}-t\left|u_{t}\right|^{2}+t^{2}\left\|u_{t}\right\|_{S_{0}^{1}}^{2} \leq t^{2} \ell\left|u_{t}\right|^{2} .
$$

Integrating between 0 and $t$ gives

$$
\left|t u_{t}\right|^{2}+\int_{0}^{t} t^{2}\left\|u_{t}\right\|_{S_{0}^{1}}^{2} d s \leq \int_{0}^{t}\left(s+\ell s^{2}\right)\left|u_{t}\right|^{2} d s .
$$

For $t=1$, we have $\sup _{s \in[0,1]}\left(s+\ell s^{2}\right)=1+\ell$. Employing this and by $(2.5)$, we have

$$
\left|u_{t}(1)\right|^{2} \leq(1+\ell) \int_{0}^{1}\left|u_{t}\right|^{2} d s \leq(1+\ell) K .
$$

Hence we obtain the uniform $L^{2}(\Omega)$ bound on $u_{t}(1)$ for all $u \in \mathcal{A}$. Since $\mathcal{A}$ is invariant, we can deduce that $-d u / d t-f(u)+g$ is uniformly bounded in $L^{2}(\Omega)$ over $\mathcal{A}$. Since $A u$ is uniformly bounded in $L^{2}(\Omega)$, we conclude that $u$ is uniformly bounded in $D(A)$ for all $u \in \mathcal{A}$. 
Remark 2.1. Using the asymptotic a priori estimate method as in [17], one can show the existence of global attractors for the semigroup $S(t)$ in the spaces $L^{p}(\Omega)$, $S_{0}^{1}(\Omega)$ and $D(A) \cap L^{2 p-2}(\Omega)$. Of course, these global attractors coincide with the global attractor $\mathcal{A}$ in $L^{2}(\Omega)$ obtained in [2].

\section{DYNAMICS OF GLOBAL ATTRACTORS}

In this section, we prove the existence of a Lyapunov function on $\mathcal{A}$ and the injectivity of the semigroup $S(t)$ on $\mathcal{A}$ using the boundedness of $\mathcal{A}$. Moreover, we show that the squeezing property for (1.1) holds.

First, we recall the concept of Lyapunov functions.

Definition 3.1. Let $S(t)$ be a continuous semigroup on a Banach space $X$. A Lyapunov function for a positively invariant set $\mathcal{M} \subset X$ is a functional $\Phi: \mathcal{M} \rightarrow \mathbb{R}$ such that

(i) $\Phi$ is continuous on $\mathcal{M}$;

(ii) $\Phi$ is nonincreasing along trajectories;

(iii) if $\Phi\left(S(t) u_{0}\right)=\Phi\left(u_{0}\right)$ for some $t>0$, then $u_{0}$ is a fixed point of $S(t)$.

We now prove the following result.

Theorem 3.1. The functional $\Phi$ on the global attractor $\mathcal{A}$ of the equation (1.1) given by

$$
\Phi(u)=\int_{\Omega}\left(\frac{1}{2}\left|\nabla_{x} u\right|^{2}+\frac{1}{2}|x|^{2 s}\left|\nabla_{y} u\right|^{2}+F(u)-g u\right) d x d y
$$

is a Lyapunov function on $\mathcal{A}$, where $F(s)=\int_{0}^{s} f(\sigma) d \sigma$.

Proof. By (2.4), we have

$$
\frac{d}{d t} \Phi(u)=-\left|u_{t}\right|^{2}
$$

which implies that $\Phi$ is strictly decreasing along the trajectories. Hence if $\Phi(u(T))=$ $\Phi\left(u_{0}\right)$ for some $T>0$, then we see that $u(t) \equiv u_{0}$ for all $0 \leq t \leq T$, and so $u_{0}$ is a fixed point. We now show the continuity of $\Phi$ on $\mathcal{A}$. Since

$$
\int_{\Omega}\left(\left|\nabla_{x} u\right|^{2}+|x|^{2 s}\left|\nabla_{y} u\right|^{2}\right) d x d y=\|u\|_{S_{0}^{1}}^{2}=(A u, u) \leq|A u||u|,
$$

we see that $\|u\|_{S_{0}^{1}}^{2}$ is Lipschitz continuous on $\mathcal{A}$. Furthermore, we have

$$
\left|\int_{\Omega}(F(u)-F(v)) d x d y\right|=\left|\int_{\Omega} \int_{v(x, y)}^{u(x, y)} f(s) d s d x d y\right|
$$




$$
\begin{aligned}
& \leq \int_{\Omega}\|f\|_{\infty}|u(x)-v(x)| d x d y \\
& \leq\|f\|_{\infty}|u-v|
\end{aligned}
$$

where $\|f\|_{\infty}:=\sup _{u \in \mathcal{A}}\|f(u)\|_{\infty}<\infty$ since $\mathcal{A}$ is bounded in $L^{\infty}(\Omega)$, and

$$
\left|\int_{\Omega}(g u-g v) d x d y\right| \leq\|g\|_{\infty}|u-v| \text { for } u, v \in \mathcal{A} .
$$

This implies that $\Phi$ is a Lyapunov function on $\mathcal{A}$.

Because the existence of a Lyapunov function, we can say more about the structure of the global attractor $\mathcal{A}$. It is known that a global attractor is the union of all bounded complete trajactories. Moreover, in our case due to the existence of a Lyapunov function, the global attractor $\mathcal{A}$ is the unstable manifold of the set of equilibria

$$
\mathcal{A}=W^{u}(E)
$$

where $E$ is the set of all fixed points of the semigroup $S(t)$.

To show the injectivity of the semigroup $S(t)$ on $\mathcal{A}$, we need the following lemma whose proof can be found in [12, Chapter 11].

Lemma 3.1. Let $H$ and $V$ be Hilbert spaces, and $H^{*}$ and $V^{*}$ be their duals, respectively, with $V \subset \subset H \cong H^{*} \subset V^{*}$. Suppose that $w \in L^{\infty}(0, T ; V) \cap L^{2}(0, T ; D(A))$ satisfies

$$
\begin{aligned}
& \frac{d w}{d t}+A w=h(t, w(t)) \text { in } L^{2}(0, T ; H), \text { and } \\
& |h(t, w(t))| \leq k(t)\|w(t)\|, \quad k(t) \in L^{2}(0, T),
\end{aligned}
$$

where $A$ is a bounded linear operator from $V$ into $V^{*}$. If $w(T)=0$, then $w(t)=0$ for all $0 \leq t \leq T$.

Theorem 3.2. The semigroup $S(t)$ has the injectivity property on $\mathcal{A}$. More precisely, if $u(t)$ and $v(t)$ are two trajectories on $\mathcal{A}$ with $u(T)=v(T)$ for some $T>0$, then $u(t)=v(t)$ for all $0 \leq t \leq T$.

Proof. Let $w=u-v$. Then we have

$$
\frac{d w}{d t}+A w+f(u)-f(v)=0
$$

Since the global attractor $\mathcal{A}$ is bounded in both $S_{0}^{1}(\Omega)$ and $D(A)$, we obtain

$$
w \in L^{\infty}\left(0, T ; S_{0}^{1}(\Omega)\right) \cap L^{2}(0, T ; D(A)) .
$$

Let

$$
h(t, w(t))=f(v(t))-f(u(t)) .
$$


Then we see that

$$
\begin{aligned}
|f(u)-f(v)|^{2} & =\int_{\Omega}|f(u)-f(v)|^{2} d x d y \\
& =\int_{\Omega}\left|\int_{v(x, y)}^{u(x, y)} f^{\prime}(s) d s\right|^{2} d x d y \\
& \leq \int_{\Omega}\left\|f^{\prime}\right\|_{\infty}^{2}|u(x, y)-v(x, y)|^{2} d x d y \\
& \leq\left\|f^{\prime}\right\|_{\infty}^{2}|u-v|_{L^{2}}^{2},
\end{aligned}
$$

where $\left\|f^{\prime}\right\|_{\infty}:=\sup _{u \in \mathcal{A}}\left\|f^{\prime}(u)\right\|_{\infty}<\infty$ since $\mathcal{A}$ is bounded in $L^{\infty}(\Omega)$. Consequently, we get

$$
|h(t, w(t))| \leq\left\|f^{\prime}\right\|_{\infty}|w(t)| \leq C\|w(t)\|_{S_{0}^{1}} .
$$

Hence we complete the proof by applying Lemma 3.1 .

By Theorem 3.2, we can see that every trajectory on $\mathcal{A}$ is defined for all $t \in \mathbb{R}$, and $S(t) \mathcal{A}=\mathcal{A}$ for all $t \in \mathbb{R}$. Hence $\{S(t)\}_{t \in \mathbb{R}}$ is a dynamical system on $\mathcal{A}$.

Definition 3.2. We say that the squeezing property holds for a semigroup $\{S(t)\}_{t \geq 0}$ if, for each $0<\delta<1$, there is a finite-rank orthogonal projection $P=P(\delta)$, with orthogonal complement $Q=Q(\delta)$, such that for all $u, v \in \mathcal{A}$, either

$$
|Q(S u-S v)| \leq|P(S u-S v)|
$$

or if not, then

$$
|S u-S v| \leq \delta|u-v|
$$

where $S=S(1)$.

We will verify that the squeezing property indeed holds for the equation (1.1). The main idea is to consider $w(t)=u(t)-v(t)$ and to estimate the equation that bound $d(P w) / d t$ below and $d(Q w) / d t$ above. The following lemma which will be used in our proof is clear.

Lemma 3.2. For $t \in[0, T]$, let $x(t) \in \mathbb{R}$ satisfy the following inequality

$$
\frac{1}{2} \frac{d}{d t}|x|^{2} \leq C(t)|x|
$$

where $C(t)$ is continuous. Then we have

$$
\frac{d}{d t}+x \mid \leq C(t)
$$

Theorem 3.3. The squeezing property holds for the semigroup $S(t)$ induced by the equation (1.1). 
Proof. Let $\omega_{j}$ be eigenfunctions of $A$ which are orthonormal in $L^{2}(\Omega)$ with corresponding eigenvalues $\lambda_{j}$, increasing in $j$ with

$$
A \omega_{j}=\lambda_{j} \omega_{j}, \lambda_{j+1} \geq \lambda_{j}, \text { for } j=1,2, \ldots
$$

(for more details, see [3]). Let $u, v \in \mathcal{A}$, and $w=u-v$. Then the equation (3.1) is satisfied. For each $n \in \mathbb{N}$, define

$$
P_{n} w=\sum_{j=1}^{n}\left(w, \omega_{j}\right) \omega_{j} \text { and } Q_{n} w=\sum_{j=n+1}^{\infty}\left(w, \omega_{j}\right) \omega_{j} .
$$

Put $p=P_{n} w$ and $q=Q_{n} w$. Then we have $w=p+q$. Take the inner product of (3.1) with $p$ to get

$$
\frac{1}{2} \frac{d}{d t}|p|^{2}+\|p\|_{S_{0}^{1}}^{2}+(f(u)-f(v), p)=0
$$

By (3.2), we have

$$
\frac{1}{2} \frac{d}{d t}|p|^{2}+\|p\|_{S_{0}^{1}}^{2} \geq-C|w||p|
$$

where $C=\left\|f^{\prime}\right\|_{\infty}$. Due to the eigenvalue expansion of $u$ and the fact that $\|u\|_{S_{0}^{1}}^{2}=$ $(A u, u)$, we have

$$
\|p\|_{S_{0}^{1}} \leq \lambda_{n}^{\frac{1}{2}}|p|
$$

and

$$
\|q\|_{S_{0}^{1}} \geq \lambda_{n+1}^{\frac{1}{2}}|q|
$$

By (3.5), we obtain

$$
\frac{1}{2} \frac{d}{d t}|p|^{2} \geq-\lambda_{n}|p|^{2}-C|w||p|
$$

By Lemma 3.2, this becomes

$$
\frac{d}{d t}+p\left|\geq-\lambda_{n}\right| p \mid-C(|p|+|q|)
$$

Similarly, we take the inner product of (3.1) with $q$ to obtain, by applying (3.6),

$$
\frac{d}{d t}|q| \leq-\lambda_{n}|q|+C(|p|+|q|)
$$

Choose $n$ sufficiently large so that

$$
\lambda_{n}-C>2 C .
$$

If (3.3) holds, then we are done. If not, we have

$$
\left|Q_{n} w(1)\right|>\left|P_{n} w(1)\right|
$$


Using (3.8), we see that

$$
\left(\lambda_{n}-C\right)\left|Q_{n} w(t)\right|>2 C\left|P_{n} w(t)\right|
$$

for $t=1$. Since $w(t)$ is continuous into $L^{2}(\Omega),(3.10)$ is satisfied in a neighborhood of $t=1$. Consider the following two cases.

Case 1. Suppose (3.10) holds for all $t \in\left[\frac{1}{2}, 1\right]$. By (3.8), we have

$$
\left(\lambda_{n}-C\right)|q|-C|p|>\frac{1}{2}\left(\lambda_{n}-C\right)|q|>C|q|
$$

for all $t \in\left[\frac{1}{2}, 1\right]$. Then (3.7) becomes

$$
\frac{d}{d t}+q\left|\leq-\lambda_{n} C\right| q \mid, \text { and so }|q(1)| \leq e^{-\frac{1}{2} \lambda_{n} C}\left|q\left(\frac{1}{2}\right)\right| \text {. }
$$

By (3.9), we get

$$
|w(1)| \leq \sqrt{2} e^{-\frac{1}{2} \lambda_{n} C}\left|q\left(\frac{1}{2}\right)\right| \leq \sqrt{2} e^{-\frac{1}{2} \lambda_{n} C}\left|w\left(\frac{1}{2}\right)\right| .
$$

Since the solutions $u$ and $v$ satisfy the Lipschitz property (see Theorem 2.4 in [2]), we obtain that

$$
\left|w\left(\frac{1}{2}\right)\right| \leq L\left(\frac{1}{2}\right)|w(0)|
$$

Hence

$$
|w(1)| \leq \sqrt{2} L\left(\frac{1}{2}\right) e^{-\frac{1}{2} \lambda_{n} C}|w(0)|
$$

This induces (3.4) if $\lambda_{n}$ is chosen to be sufficiently large enough.

Case 2. Suppose there exists $t_{0} \geq 1 / 2 \operatorname{such}$ that $(3.10)$ is valid on $\left(t_{0}, 1\right]$ and

$$
\left(\lambda_{n}-C\right)\left|Q_{n} w\left(t_{0}\right)\right|=2 C\left|P_{n} w\left(t_{0}\right)\right|
$$

Define a map $\Phi:\left[t_{0}, 1\right] \rightarrow \mathbb{R}$ by

$$
\Phi(t)=\Phi(p(t), q(t))=(|p|+|q|) \exp \left(\frac{\lambda_{n}|q|}{C(|p|+|q|)}\right) .
$$

Then we have

$$
\frac{d \Phi}{d t} \leq 0 \text { for all } t \in\left[t_{0}, 1\right]
$$

Hence

$$
\Phi(1) \leq \Phi\left(t_{0}\right)
$$

Since (3.10) holds at $t=1$, we obtain

$$
\Phi(1) \geq|q(1)| e^{\lambda_{n} / C} .
$$


On the other hand, since (3.11) holds at $t=t_{0}$, it follows that

$$
2 C\left(\left|p\left(t_{0}\right)\right|+\left|q\left(t_{0}\right)\right|\right)=\left(\lambda_{n}+C\right)\left|q\left(t_{0}\right)\right|,
$$

and so

$$
\Phi\left(t_{0}\right)=\frac{\lambda_{n}+C}{2 C}\left|q\left(t_{0}\right)\right| e^{2 \lambda_{n} /\left(\lambda_{n}+C\right)} .
$$

Consequently

$$
|q(1)| \leq e^{-\lambda_{n} / C} \frac{\lambda+C}{2 C} e^{2}\left|q\left(t_{0}\right)\right| .
$$

Using the Lipschitz property of the solutions again, we see that

$$
|q(1)| \leq e^{-\lambda_{n} / C} \frac{\lambda+C}{2 C} e^{2} L(1)|w(0)| .
$$

Since $|p(1)|<|q(1)|$, we must have

$$
|w(1)| \leq 2 e^{-\lambda_{n} / C} \frac{\lambda_{n}+C}{2 C} e^{2} L(1)|w(0)| .
$$

Hence if we choose $n$ sufficiently large enough, we obtain (3.4), and so it completes the proof.

Remark 3.1. As pointed out in [12, Chapter 14], the squeezing property has many important consequences. First, using the squeezing property one can show that the global attractor $\mathcal{A}$ lies close to a finite-dimensional Lipschitz manifold as a graph over $P L^{2}(\Omega)$ and that the dynamics "projected" onto $P L^{2}(\Omega)$ (here $P=P(\delta)$ is the finite-rank orthogonal projection in Definition 3.2) give a good indication on the asymptotic behavior of solutions. Furthermore, one can prove that the flow on the global attractor $\mathcal{A}$ has a finite number of determining modes, i.e., for two solutions $u(t)$ and $v(t)$ on the the attractor, if

$$
|P(u(t)-v(t))| \rightarrow 0 \quad \text { as } \quad t \rightarrow \infty
$$

then

$$
|u(t)-v(t)| \rightarrow 0 \quad \text { as } \quad t \rightarrow \infty
$$

\section{FRACTAL DIMENSION ESTIMATES}

In this section, we obtain an upper bound and a lower bound of the fractal dimension of the global attractor $\mathcal{A}$. For brevity, we denote $V=S_{0}^{1}(\Omega)$ and $H=L^{2}(\Omega)$. The scalar product on $V$ and $H$ will be denoted by $((\cdot, \cdot))$ and $(\cdot, \cdot)$, respectively. Then we have

$$
V \subset H \cong H^{*} \subset V^{*}
$$


Let $F(u)=-G_{s}+f(u)-g$, where $u$ is the solution of (1.1), $g \in L^{\infty}(\Omega)$, and $f: \mathbb{R} \rightarrow \mathbb{R}$ is a function of polynomial type satisfying (1.2)-(1.3) and an additional assumption:

$$
f \in C^{2}(\mathbb{R}) \text { and } f^{\prime \prime} \text { is uniformly bounded on the bounded sets of } \mathbb{R} \text {. }
$$

For any $t \in[0, T]$, define

$$
A(t): V \rightarrow V^{*} \text { by } A(t)=F^{\prime}(u(t))
$$

where $F^{\prime}$ is the Fréchet derivative of $F$ with $F^{\prime}(u)=-G_{s}+f^{\prime}(u)$. Then $A(t)$ is an isomorphism sending $D(A(t))$ onto $H$. For each $t \in[0, T]$, define $a(t ; \cdot, \cdot): V \times V \rightarrow \mathbb{R}$ by

$$
a(t ; u, v)=\langle A(t) u, v\rangle,
$$

where $\langle\cdot, \cdot\rangle$ is a dual pairing of $V$ and $V^{*}$. Then $\{a(t ; u, v): t \in[0, T]\}$ is a family of bilinear continuous forms on $V$ such that

$$
\begin{aligned}
& \forall u, v \in V, t \mapsto a(t ; u, v) \text { is a measurable function, } \\
& \exists M=M_{T}<\infty \text { s.t. }|a(t ; u, v)| \leq M_{T}\|u\|\|v\|, \forall u, v \in V \text {, a.e. } t \in[0, T], \\
& \exists \alpha>0 \text { s.t. } a(t ; u, u) \geq \alpha\|u\|^{2}, u \in V \text {, a.e. } t \in[0, T] .
\end{aligned}
$$

For any $\xi \in H$, consider the following initial value problem

$$
\left\{\begin{array}{l}
\frac{d U(t)}{d t}+A(t) U(t)=0 \text { on }(0, T) \\
U(0)=\xi
\end{array}\right.
$$

Then from (4.2)-(4.4) we can show that there exists a unique solution $U$ of (4.5) such that

$$
\begin{aligned}
& U \in L^{2}(0, T ; V) \cap C([0, T] ; H), \\
& U^{\prime} \in L^{2}\left(0, T ; V^{\prime}\right) .
\end{aligned}
$$

From the above setting, we have the following result on the uniform differentiability of $S(t)$ on $\mathcal{A}$.

Lemma 4.1. For every $t>0, S(t)$ is uniformly differentiable on $\mathcal{A}$. Its differential at $u_{0} \in \mathcal{A}$ is the linear operator $L\left(t, u_{0}\right): \xi \in H \mapsto U(t) \in H$, where $U(\cdot)$ is the solution of (4.5). Moreover, we have $\sup _{u_{0} \in \mathcal{A}}\left|L\left(t, u_{0}\right)\right|_{\mathscr{L}(H)}<\infty$.

Proof. Let $t_{0}>0$ and $u_{0}, v_{0} \in \mathcal{A}$. Let $u, v$ be the solutions of (1.1) with $u(0)=u_{0}$ and $v(0)=v_{0}$, and $U$ be the solution of (4.5) with $\xi=u_{0}-v_{0}$. For each $t \geq 0$, define a linear operator

$$
L\left(t, u_{0}\right): \xi \in H \mapsto U(t) \in H .
$$


Let $\eta(t)=v(t)-u(t)-U(t)$ for $t \geq 0$. Then we see that $\eta(t)$ satisfies $\eta(0)=0$ and

$$
\frac{d \eta}{d t}-G_{s} \eta+f^{\prime}(u) \eta=h
$$

where

$$
h=f(u)-f(v)+f^{\prime}(u)(v-u)=\left[f^{\prime}(u)-f^{\prime}(u+\theta(v-u))\right](u-v), \quad 0<\theta<1 .
$$

By Theorem 2.1 and (4.1), we see that

$$
|h(t)| \leq c|u-v|^{2}
$$

for some $c>0$ and all $t \geq 0$. Hence, by Theorem 2.4 in [2], we get

$$
|h(t)| \leq c e^{4 \ell t}\left|u_{0}-v_{0}\right|^{2}, \text { for all } t \geq 0 .
$$

We multiply (4.8) by $\eta$ and integrate over $\Omega$. Then (1.3) and Green's formula yield

$$
\frac{1}{2} \frac{d}{d t}|\eta|^{2}+\|\eta\|_{S_{0}^{1}}^{2} \leq \ell|\eta|^{2}+\int_{\Omega} h \eta d x d y \leq\left(\ell+\frac{1}{2}\right)|\eta|^{2}+\frac{1}{2}|h|^{2} .
$$

Hence by (4.9),

$$
\frac{d}{d t}|\eta|^{2} \leq(2 \ell+1)|\eta|^{2}+c^{2} e^{8 \ell t_{0}}\left|u_{0}-v_{0}\right|^{4}, \text { for all } 0 \leq t \leq t_{0} .
$$

By Gronwall's inequality, we obtain

$$
\left|\eta\left(t_{0}\right)\right|^{2} \leq \frac{e^{(2 \ell+1) t_{0}}-1}{2 \ell+1} c^{2} e^{8 \ell t_{0}}\left|u_{0}-v_{0}\right|^{4} .
$$

This implies that $S\left(t_{0}\right)$ is uniformly differentiable on $\mathcal{A}$, and its differential at $u_{0} \in \mathcal{A}$ is $L\left(t_{0}, u_{0}\right)$. Moreover, by the continuous dependence of solution of (4.5) on the initial data, we can see that $\sup _{u_{0} \in \mathcal{A}}\left|L\left(t, u_{0}\right)\right|_{\mathscr{L}(H)}<\infty$.

We now recall the definition of fractal dimension. The fractal dimension of $\mathcal{A}$ is defined by

$$
d_{F} \mathcal{A}=\limsup _{\varepsilon \rightarrow 0} \frac{\log N(\mathcal{A}, \varepsilon)}{\log (1 / \varepsilon)}
$$

where $N(\mathcal{A}, \varepsilon)$ is the minimum number of the balls of radius $\varepsilon>0$ to cover $\mathcal{A}$.

For $L \in \mathscr{L}(H)$ and $m \in \mathbb{N}$, let $\omega_{m}(L)$ be the norm of the exterior product $\wedge^{m} L$ in $\wedge^{m} H$, that is,

$$
\omega_{m}(L)=\sup _{\substack{\xi_{1}, \cdots, \xi_{m} \in H \\\left|\xi_{i}\right| \leq 1}}\left|L \xi_{1} \wedge \cdots \wedge L \xi_{m}\right|
$$

For each $m \in \mathbb{N}$, let

$$
\bar{\omega}_{m}(t)=\sup _{u_{0} \in \mathcal{A}} \omega_{m}\left(L\left(t, u_{0}\right)\right), t \geq 0, \text { and } \Pi_{m}=\lim _{t \rightarrow \infty} \bar{\omega}_{m}(t)^{1 / t} .
$$


The uniform Lyapunov numbers, $\mu_{m}$ are given by

$$
\mu_{1}=\log \Pi_{1}, \mu_{m}=\log \Pi_{m}-\log \Pi_{m-1}, m \geq 2 .
$$

To estimate the fractal dimension of the global attractor, we use the following abstract result.

Lemma 4.2. [9, Theorem 3.1] If $\mu_{1}+\cdots+\mu_{m}<0$ for some $m \in \mathbb{N}$, then

$$
d_{F} \mathcal{A} \leq m \max _{1 \leq j \leq m-1}\left(1+\frac{\left(\mu_{1}+\cdots+\mu_{j}\right)_{+}}{\left|\mu_{1}+\cdots+\mu_{m}\right|}\right) .
$$

The crucial step to obtain an upper bound of the fractal dimension of $\mathcal{A}$ is to estimate the Lyapunov numbers of $\mathcal{A}$.

Theorem 4.1. The fractal dimension of $\mathcal{A}$ is less than or equal to $2 m$, where $m \in \mathbb{N}$ is such that

$$
m-1<\left(\frac{2 \kappa_{2}}{\kappa_{1}}\right)^{N(s) /(2+N(s))} \leq m .
$$

Here $\kappa_{1}=\frac{1}{2} c_{1}, \kappa_{2}=c_{3}\left(c_{2}+\ell\right)^{1+(N(s) / 2)}$ for some constants $c_{1}, c_{2}$ and $c_{3}$. More precisely, $c_{1}, c_{2}$ and $c_{3}$ are obtained in the proof below.

Proof. Note that

$$
\mu_{1}+\cdots+\mu_{m}=\log \Pi_{m}, \text { for } m \in \mathbb{N} .
$$

According to (4.10), we obtain

$$
\omega_{m}\left(L\left(t, u_{0}\right)\right)=\sup _{\substack{\xi_{i} \in H \\\left|\xi_{i}\right| \leq 1}}\left|U_{1} \wedge \cdots \wedge U_{m}\right|
$$

where $U_{i}$ is the solution of (4.5) with $U_{i}(0)=\xi_{i}$ for $1 \leq i \leq m$. By Lemma 1.2 in [13, Chapter V], we see that

$$
\frac{1}{2} \frac{d}{d t}\left|U_{1} \wedge \cdots \wedge U_{m}\right|^{2}=-\operatorname{Tr}\left(F^{\prime}(u) \circ Q_{m}\right)\left|U_{1} \wedge \cdots \wedge U_{m}\right|^{2},
$$

where $Q_{m}$ is the orthogonal projection in $H$ onto the space spanned by $U_{1}, \cdots, U_{m}$. So we get

$$
\omega_{m} L\left(t, u_{0}\right) \leq \sup _{\substack{\xi_{i} \in H \\\left|\xi_{i}\right| \leq 1}} \exp \left(-\int_{0}^{t} \operatorname{Tr} F^{\prime}\left(S(\tau) u_{0}\right) \circ Q_{m}(\tau) d \tau\right)
$$

For each $m \in \mathbb{N}$, we let

$$
q_{m}=\limsup _{t \rightarrow \infty}\left\{\inf _{u_{0} \in \mathcal{A}} \frac{1}{t} \int_{0}^{t} \inf _{\operatorname{rank} Q=m} \operatorname{Tr} F^{\prime}\left(S(\tau) u_{0}\right) \circ Q(\tau) d \tau\right\},
$$


where $Q$ is any orthogonal projection in $H$ with $Q H \subset V$. From (4.11), we get

$$
\bar{\omega}_{m}(t)=\sup _{u_{0} \in \mathcal{A}} \omega_{m}\left(L\left(t, u_{0}\right)\right) \leq \sup _{u_{0} \in \mathcal{A}} \exp \left\{-\int_{0}^{t} \inf _{\operatorname{rank} Q=m} \operatorname{Tr} F^{\prime}\left(S(\tau) u_{0}\right) \circ Q(\tau) d \tau\right\} .
$$

Hence we obtain

$$
\Pi_{m}=\lim _{t \rightarrow \infty}\left\{\bar{\omega}_{m}(t)\right\}^{1 / t} \leq \exp \left(-q_{m}\right) .
$$

Consequently, if $q_{m}>0$ for some $m$, then by Lemma 4.2 , we get

$$
d_{F} \mathcal{A} \leq m \max _{1 \leq j \leq m-1}\left\{1+\frac{\left(-q_{j}\right)_{+}}{q_{m}}\right\} .
$$

We now show that $q_{m}>0$ for some $m$. Let $Q$ be an orthogonal projection in $H$ of rank $m$ with $Q H \subset V$. Let $\left\{\phi_{j}\right\}_{j \in \mathbb{N}}$ be an orthonormal basis of $H$ with $\phi_{1}, \ldots, \phi_{m}$ being a basis of $Q H$. Then we have

$$
\begin{aligned}
\operatorname{Tr} F^{\prime}(u) \circ Q=\sum_{j=1}^{m}\left(F^{\prime}(u) \phi_{j}, \phi_{j}\right) & =\sum_{j=1}^{m}\left\|\phi_{j}\right\|_{S_{0}^{1}}^{2}+\int_{\Omega} f^{\prime}(u) \rho d x d y \\
& \geq \sum_{j=1}^{m}\left\|\phi_{j}\right\|_{S_{0}^{1}}^{2}-\ell \int_{\Omega} \rho d x d y
\end{aligned}
$$

where

$$
\rho(x, y)=\sum_{j=1}^{m}\left|\phi_{j}(x, y)\right|^{2}
$$

Let $\left\{\lambda_{j}\right\}$ be the sequence of eigenvalues of $A$ in $L^{2}(\Omega)$ such that $0<\lambda_{1} \leq \lambda_{2} \leq \cdots$. Then we see that

$$
\sum_{j=1}^{m}\left\|\phi_{j}\right\|_{S_{0}^{1}}^{2} \geq \lambda_{1}+\cdots+\lambda_{m}
$$

Moreover, we have

$$
\lambda_{1}+\cdots+\lambda_{m} \geq c_{1} m^{1+(2 / N(s))}-c_{2} m,
$$

where $c_{1}=c_{1}\left(N, s,|\Omega|, \alpha_{N-1}\right)$ is a constant depending on $N, s,|\Omega|$ and $\alpha_{N-1}$, and $c_{2}=c_{2}(s)$ is a constant depending on $s$. Here $\alpha_{N-1}$ is the area of the unit sphere in $\mathbb{R}^{N}$ (for more details, see Theorem 1.1 in [3]). Hence we get

$$
\sum_{j=1}^{m}\left\|\phi_{j}\right\|_{S_{0}^{1}}^{2} \geq c_{1} m^{1+(2 / N(s))}-c_{2} m
$$

Since each $\phi_{j}$ is mutually orthonormal, we have

$$
\operatorname{Tr} F^{\prime}(u) \circ Q \geq c_{1} m^{1+(2 / N(s))}-m\left(c_{2}+\ell\right),
$$

where $\int_{\Omega} \rho d x d y=m$. By Young's inequality, we obtain

$$
m\left(c_{2}+\ell\right) \leq \frac{1}{2} c_{1} m^{1+(2 / N(s))}+c_{3}\left(c_{2}+\ell\right)^{1+(N(s) / 2)},
$$


where $c_{3}$ depends on $N, s,|\Omega|$ and $\alpha_{N-1}$. Consequently, we get

$$
\operatorname{Tr} F^{\prime}(u) \circ Q_{\geq} \frac{1}{2} c_{1} m^{1+(2 / N(s))}-c_{3}\left(c_{2}+\ell\right)^{1+(N(s) / 2)} .
$$

Hence by (4.12), we have

$$
q_{m} \geq \kappa_{1} m^{1+(2 / N(s))}-\kappa_{2},
$$

where $\kappa_{1}=\frac{1}{2} c_{1}$ and $\kappa_{2}=c_{3}\left(c_{2}+l\right)^{1+(N(s) / 2)}$. Let $m \in \mathbb{N}$ be such that

$$
q_{m}>0 \text { and } m-1<\left(\frac{2 \kappa_{2}}{\kappa_{1}}\right)^{N(s) /(2+N(s))} \leq m .
$$

Then we obtain

$$
\frac{\left(-q_{j}\right)_{+}}{q_{m}} \leq 1, \quad \forall j=1, \ldots, m-1 .
$$

Hence we conclude that $d_{F} \mathcal{A} \leq 2 m$ from (4.13).

We now find a lower bound of the fractal dimension of the global attractor $\mathcal{A}$ induced by the equation (1.1). For simplicity, we assume $g \equiv 0$ and $f(0)=0$ in the equation (1.1). Then we can see the following properties:

- the mapping $\left(t, u_{0}\right) \mapsto S(t) u_{0}$ from $\mathbb{R}_{+} \times H$ into $H$ is continuous;

- $\mathbf{0} \in H$ is a fixed point of $S(t)$.

Moreover, we can check that $S(t)$ is Fréchet differentiable at $\mathbf{0}$ for all $t \in \mathbb{R}_{+}$with differential $S^{\prime}(t)(\mathbf{0})=L(t, \mathbf{0})$. Let us write $S^{\prime}(t)=S^{\prime}(t)(\mathbf{0})$ for simplicity. Then we can show that the spectrum of $S^{\prime}(t)$ consists of its eigenvalues and 0 . To show this, let us consider the following first variation equation which is obtained from (4.5) by putting $u=\mathbf{0}$ :

$$
\left\{\begin{array}{l}
U_{t}-G_{s} U+f^{\prime}(0) U=0 \\
U(0)=\xi
\end{array}\right.
$$

Then we have

$$
-G_{s} w_{k}=\lambda_{k} w_{k}, \quad w_{k} \in S_{0}^{1}(\Omega),
$$

and may assume that

$$
0<\lambda_{1} \leq \lambda_{2} \leq \cdots, \quad \lambda_{k} \rightarrow \infty \text { as } k \rightarrow \infty
$$

Without loss of generality, we can suppose that $f^{\prime}(0)+\lambda_{k} \neq 0$ for all $k \in \mathbb{N}$. Put $\mu_{k}=f^{\prime}(0)+\lambda_{k}$. Then we obtain

$$
-G_{s} w_{k}+f^{\prime}(0) w_{k}=\mu_{k} w_{k}
$$


Consequently, we see that the eigenvalues of $S^{\prime}(t)$ are the numbers $e^{-\mu_{k} t}$, and the corresponding eigenfunctions are $w_{k}$ for $k \in \mathbb{N}$. If $\lambda \notin\left\{e^{-\mu_{k} t}, 0: k \in \mathbb{N}\right\}$, then $S^{\prime}(t) \zeta-\lambda \zeta \neq \mathbf{0}$ for $\zeta \in H \backslash\{\mathbf{0}\}$. This means that the spectrum of $S^{\prime}(t)$ consists of eigenvalues and 0 .

Let $n$ be the number of $\lambda_{k}^{\prime}$ s with $\lambda_{k}<-f^{\prime}(0)$, and let $H_{+}$be the subspace of $H$ spanned by the eigenvectors corresponding to the eigenvalues $e^{-\mu_{k} t}$ of $S^{\prime}(t)$ with $\mu_{k}<0$. Then we know that $\operatorname{dim} H_{+}=n$. This shows that $z=\mathbf{0}$ is a hyperbolic fixed point of $S^{\prime}(t)$ since the spectrum of $S^{\prime}(t)$ does not intersect with the unit circle. Moreover, we see that $S^{\prime}(t)$ satisfies a Lipschitz condition:

$$
\left\|S^{\prime}(t) u_{1}-S^{\prime}(t) u_{2}\right\| \leq\left\|u_{1}-u_{2}\right\| \text { for } u_{1}, u_{2} \in H .
$$

For $r>0$, we let

$$
W_{+}^{r}(z)=\left\{u_{0} \in B_{r}(z): \exists u_{n} \in H \text { s.t. } S(n) u_{n}=u_{0} \text { and } u_{n} \rightarrow z \text { as } n \rightarrow \infty\right\},
$$

where $B_{r}(z)$ is the ball of radius $r$ centered at $z$ under a suitable norm on $H$. Then we have that $W_{+}^{r}(z) \subset \mathcal{A}$ for sufficiently small $r>0$, and $W_{+}^{r}(z)$ is a $C^{1}$ manifold of dimension equal to $\operatorname{dim} H_{+}=n$. Consequently, we have proved the following theorem.

Theorem 4.2. The fractal dimension $d_{F} \mathcal{A}$ of the global attractor $\mathcal{A}$ of the equation (1.1) is greater than or equal to $n$, where $n=\operatorname{dim} H_{+}$.

\section{ACKNOWLEDGEMENTS}

This work is supported by NRF Grant No. NRF-20151009350 and NRF-2014R1A1A2 056839 .

\section{REFERENCES}

[1] C.T. Anh, Pullback attractor for a non-autonomous parabolic equation involving Grushin operators, Electronic J. Differential Equations (2010), No 11, 1-14.

[2] C.T. Anh and T.D. Ke, Existence and continuity of global attractor for a semilinear degenerate parabolic equation, Electronic J. Differential Equations (2009), No 61, 1-13.

[3] H. Chen and P. Luo, Lower bounds of Dirichlet eigenvalues for some degenerate elliptic operators, Calc. Var. 54 (2015), 2831-2852.

[4] L. D'Ambrosio, Hardy inequalities related to Grushin type operators, Proc. Amer. Math. Soc. 132 (2003), 725-734. 
[5] L. D'Ambrosio and S. Lucente, Nonlinear Liouville theorems for Grushin and Tricomi operators, J. Differential Equations 193 (2003), 511-541.

[6] B. Franchi and E. Lanconelli, An embedding theorem for Sobolev spaces related to nonsmooth vector fields and Harnack inequality, Comm. Partial Differential Equations 9 (1984), 1237-1264.

[7] V.V. Grushin, A certain class of elliptic pseudo differential operators that are degenerated on a submanifold, Mat. Sb. 84 (1971), 163-195; English translation in: Math. USSR Sbornik 13 (1971), 155-183.

[8] A.E. Kogoj and E. Lanconelli, On semilinear $\Delta_{\lambda}$-Laplace equation, Nonlinear Anal. 75 (2012), 4637-4649.

[9] M. Marion, Attractors for reaction-diffusion equations: existence and estimate of their dimension, Appl. Anal. 25 (1987), 101-147.

[10] R. Monti and D. Morbidelli, Kelvin transform for Grushin operators and critical semilinear equations, Duke Math. J. 131 (2006), 167-202.

[11] D.D. Monticelli, Maximum principles and the method of moving planes for a class of degenerate elliptic linear operators, J. Eur. Math. Soc. (JEMS) 12 (2010), 611-654.

[12] J.C. Robinson, Infinite-Dimensional Dynamical Systems: An Introduction to Dissipative Parabolic PDEs and the Theory of Global Attractors, Cambridge Texts in Applied Mathematics, Cambridge University Press, Cambridge (2001).

[13] R. Temam, Infinite Dimensional Dynamical Systems in Mechanic and Physics, 2nd edition, Springer-Verlag, New York (1997).

[14] N.T.C. Thuy and N.M. Tri, Existence and nonexistence results for boundary value problems for semilinear elliptic degenerate operator, Russian J. Math. Phys. 9 (2002), 366-371.

[15] Q. Yang, D. Su and Y. Kong, Improved Hardy inequalities for Grushin operators, J. Math. Anal. Appl. 424 (2015), 321-343.

[16] X. Yu, Liouville type theorem for nonlinear elliptic equation involving Grushin operators, Commun. Contemp. Math. 17 (2015), 1450050, 12 pp.

[17] C.K. Zhong, M.H. Yang and C. Sun, The existence of global attractors for the norm-to-weak continuous semigroup and application to the nonlinear reactiondiffusion equations, J. Differential Equations 223 (2006), 367-399. 